\title{
Cerebrospinal fluid anti-myelin antibodies are related to magnetic resonance measures of disease activity in multiple sclerosis
}

\author{
M H J Vogt, ${ }^{1}$ C E Teunissen, ${ }^{1}$ E lacobaeus, ${ }^{2}$ D A M Heijnen,, E C W Breij,, ${ }^{1}$ T Olsson, ${ }^{2}$ \\ L Brundin, ${ }^{2}$ J Killestein, ${ }^{3}$ Christine D Dijkstra ${ }^{1}$
}

${ }^{1}$ Department of Molecular Cell Biology and Immunology, VU University Medical Centre, Amsterdam, The Netherlands;

${ }^{2}$ Department of Clinical Neuroscience,

Neuroimmunology Unit, Karolinska Institute, Stockholm Sweden; ${ }^{3}$ Department of Neurology, VU University Medical Centre, Amsterdam The Netherlands

Correspondence to: Dr C D Dijkstra, Department of Molecular Cell Biology and Immunology, VU University Medical Centre, PO Box 7057 1007 MB Amsterdam, The Netherlands; cd.dijkstra@ vumc.nl

Received 6 February 2008 Revised 30 June 2008 Accepted 13 July 2008 Published Online First 23 October 2008

\section{ABSTRACT}

Objective: Recent studies reported contrasting results with respect to the presence of anti-myelin protein antibodies in multiple sclerosis (MS) and their relation with disease activity. This may be due to the heterogeneous specificity of autoantibodies in MS and the inability of most methods to detect pathogenically relevant antibodies. Here, myelin particles were used to detect anti-myelin antibodies in the CSF of MS patients. Subsequently, their relation with MRI parameters was evaluated

Methods: Anti-myelin IgG antibody reactivity was determined in the CSF of patients with MS $(n=65)$ and clinically isolated syndrome (CIS, $n=37$ ) using a novel flow cytometry based assay. In addition, the CSF of patients with other neurological diseases (OND, $n=17$ ), inflammatory neurological diseases (IND, $n=33$ ) and controls ( $\mathrm{n}=22$ ) was tested.

Results: Compared with controls, increased anti-myelin $\mathrm{IgG}$ antibody reactivity was most frequently found in the CSF of patients with CIS $(46 \%, p=0.002)$, relapsingremitting MS $(56 \%, p<0.001)$ and secondary progressive MS (55\%, $p<0.001)$, together constituting $85 \%$ of all positive CSF samples. In contrast, elevated anti-myelin IgG antibody reactivity was present in a minority of IND patients (21\%), marginally present in controls $(5 \%)$ and absent in OND patients (0\%). Most strikingly, anti-myelin $\lg \mathrm{G}$ antibody reactivity was related to the number of $\mathrm{T} 2$ lesions ( $r=0.31, p=0.041)$ and gadolinium enhancing $\mathrm{T1}$ lesions ( $r=0.37, p=0.016$ ) on brain MRI in CIS and relapse onset MS patients.

Conclusion: CSF anti-myelin $\lg \mathrm{G}$ antibodies are promising specific biomarkers in CIS and relapse onset MS and correlate with MR measures of disease activity.

The pathogenesis of multiple sclerosis (MS) involves the immune mediated degradation of myelin, the fatty sheath that surrounds the nerve fibres of the CNS. ${ }^{1}$ Several studies have suggested that humoral immunity plays a role in the pathogenesis of MS. Antibody deposition in MS has been found to be associated with disintegration of myelin around axons in lesions ${ }^{2}$ and transfer of anti-myelin specific antibodies to animals with experimental autoimmune encephalomyelitis resulted in a more severe disease course. ${ }^{34}$ The pathogenic role of antibodies in the demyelination process involves activation of complement and receptor mediated phagocytosis of myelin by macrophages. ${ }^{56}$

Analysis of the pathology of MS lesions revealed that in approximately $50 \%$ of MS patients, the demyelination process showed close similarities to antibody mediated autoimmune encephalomyelitis. ${ }^{7}$ These patients are also more likely to respond to therapeutic plasma exchange compared with patients with other pathological patterns. ${ }^{8}$ However, clinical studies that characterised antibody responses in MS demonstrated contrasting results with respect to the frequency of autoantibody positive MS patients. Myelin oligodendrocyte glycoprotein (MOG) specific antibodies have been detected in MS patients at variable frequencies, ranging from as low as $6 \%$ to $38 \% .{ }^{4}{ }^{10}$ Recently, O'Connor et al reported that anti-MOG antibodies were only present in acute disseminated encephalomyelitis and almost undetectable in adult onset MS patients. ${ }^{11}$ Similar contrasting results have been obtained with respect to autoantibody levels and MS disease activity and progression. Kuhle et al recently reported that there was no association between the presence of serum anti-MBP and antiMOG specific antibodies in patients who had a clinically isolated syndrome (CIS) and their risk of developing clinically definite $\mathrm{MS},{ }^{12}$ as was suggested by a previous study. ${ }^{13}$ Another recent study in a second CIS cohort of the same authors confirmed the lack of prognostic value of these antibodies for clinically definite MS. ${ }^{14}$ However, patients with anti-MOG and anti-MBP antibodies had higher lesion load and more disseminated lesions in cranial MRI, which was in contrast with a previous study that demonstrated no association between anti-MOG and anti-MBP antibodies and MRI findings at baseline and follow-up. ${ }^{15}$

A common problem encountered in these clinical studies is that accurate detection of pathogenically relevant antibodies requires a properly folded and post-translationally modified target protein. ${ }^{16-18}$ Recombinant, in vitro translated and native purified myelin proteins have been used for detection of myelin specific antibodies. ${ }^{13}{ }^{18-20}$ None of these methods takes into account the specific structure of folded myelin proteins in association with a hydrophobic, lipid-rich bilayer membrane as they are displayed to the immune system in vivo on myelin sheaths. Additionally, antibody responses in MS are not restricted to a single myelin protein but show a heterogeneous specificity, including reactivity to myelin lipids. ${ }^{21}$ Therefore, autoantibody responses in MS may only be partly or not at all detected by determination of reactivity to a single myelin protein.

Recently, we described a novel flow cytometry based assay that detects antibodies that bind to 
myelin particles, which have a bead-like appearance in aqueous solution. ${ }^{22}$ The assay allows the simultaneous detection of antimyelin antibodies specific for a wide variety of myelin proteins and lipids. In addition, myelin proteins and lipids are displayed to the antibodies in a similar manner as they are presented in vivo on an initial immune response. Here we demonstrate that CSF anti-myelin IgG antibodies are increased in half of CIS patients and in the majority of relapse onset MS patients whereas they were absent in the CSF of controls and patients with other neurological diseases. Most strikingly, the presence of anti-myelin IgG antibodies in the CSF of CIS and relapse onset MS patients was related to the number of $\mathrm{T} 2$ and gadolinium enhancing T1 lesions on brain MRI.

\section{MATERIALS AND METHODS}

\section{Isolation of human myelin}

Human myelin was isolated as described previously. ${ }^{22}$ Briefly, CNS white matter was homogenised in $0.32 \mathrm{M}$ sucrose. The suspension was layered over a $0.85 \mathrm{M}$ solution of sucrose and centrifuged at $75000 \mathrm{~g}$ for $30 \mathrm{~min}$ at $4^{\circ} \mathrm{C}$ (step 1). The interphase, containing the myelin, was pooled and washed three times with deionised water by repeated centrifugation (75 $000 \mathrm{~g}, 15 \mathrm{~min}, 4^{\circ} \mathrm{C}$ ) (step 2). Steps 1 and 2 were repeated twice. Myelin was collected and stored at $-20^{\circ} \mathrm{C}$ until further use.

The protein concentration of the myelin preparation was calculated using a bovine serum albumin standard curve, as described previously. ${ }^{23}$ Myelin from five subjects was pooled and used in all assays.

\section{Myelin flow cytometry assay}

We used the myelin flow cytometry assay, described previously ${ }^{22}$. Briefly, human myelin $(15 \mu \mathrm{g})$ and undiluted CSF $(15 \mu \mathrm{l})$ were added to $100 \mu \mathrm{l}$ of phosphate buffered saline (PBS, $\mathrm{pH} 7.4$ ) in a 96 well V-bottom plate and incubated for $30 \mathrm{~min}$ at $37^{\circ} \mathrm{C}$. Unbound CSF proteins were removed by washing the myelin in PBS (4500 rpm, 4 min; 3×). Myelin particles were subsequently incubated with biotinylated goat anti-human IgG (Jackson Laboratories, West Grove, Pennsylvania, USA) for $30 \mathrm{~min}$ at $37^{\circ} \mathrm{C}$. After washing in $\mathrm{PBS}$, samples were incubated with Alexa $^{488 / 594}$ conjugated streptavidin (Molecular Probes, US), washed again and taken up in $50 \mu \mathrm{l}$ of PBS in FACS tubes (B\&D Biosciences, Franklin Lakes, New Jersey, USA). Myelin immunoreactivity was measured using the FACS Calibur (B\&D Biosciences). Anti-myelin antibody levels were expressed as mean fluorescence intensity (MFI). All CSF samples were incubated with myelin in triplicate and all samples were included in at least three separate experiments.

\section{Patient and control characteristics}

CSF samples were obtained from a CSF library stored at the Neurological Clinic, Karolinska Hospital, KS. The library contains CSF from patients having undergone diagnostic lumbar punctures at the Neurological Clinic Karolinska Hospital between 2001 and 2006. The majority of patients participated in the STOP MS project. The samples have been stored coded and made anonymous in accordance with the Medical Research Council guidelines on the ethical use of biological specimen collections in clinical research.

In all MS patients $(n=65)$, definite $M S$ was diagnosed according to the criteria of McDonald et al by trained neurologists. ${ }^{24}$ Patients were classified as having a relapsing remitting (RRMS, $n=36$ ) or secondary progressive (SPMS, $\mathrm{n}=29) \mathrm{MS}$ disease course according to Lublin and Reingold. ${ }^{25} \mathrm{~A}$ trained neurologist scored the symptoms and signs of MS. Disability was measured using the Expanded Disability Status Scale (EDSS). Disease activity was based on clinical evaluation. Exacerbation was defined as a decline in neurological status $\geqslant 1$ grade on the EDSS, the symptoms of which had started more than a week prior to sampling. Patients whose neurological deficits had remained stable for the last month were regarded as being in remission. CIS patients $(n=37)$ had a first clinical episode with one or more MRI lesions characteristic of MS

MRI data obtained within 2 months of CSF sampling were included in this study $(n=52)$. The routine MRI examinations were performed on a $1.5 \mathrm{~T}$ GE scanner with a birdcage head coil (Sigma Lx or Excite) using the following sequences: sagittal and axial fast spin echo (FSE), FLAIR, with $5 \mathrm{~mm}$ slices (no gaps) or $4 \mathrm{~mm}$ slices, respectively (TE/TR/TF 140/9000/12000). Axial and sagittal fast spin echo T2 (TE/TR/echo train length $=85 /$ 4500/11-12). Pre- and postcontrast axial, spin echo T1 (TE/TR 10/560) $5 \mathrm{~mm}$ slices with $2 \mathrm{~mm}$ gaps and $4 \mathrm{~mm}$ slices without gaps, respectively. Post-contrast images approximately 5-6 min after $0.1 \mathrm{mM} / \mathrm{kg}$ bodyweight Ominscan (GE Medical).

Isoelectric focusing with immunoblotting on agarose gels was used for the detection of oligoclonal bands in CSF and serum samples.

Three control groups were used. One group consisted of patients with other neurological diseases (OND, $\mathrm{n}=17$ ), such as normal pressure hydrocephalus $(n=3)$, brain tumours $(n=3)$, migraine $(n=2)$, headache $(n=2)$, vertebral artery dissection $(n=1)$, polyneuropathy $(n=1)$, neuralgia $(n=1)$, myasthenia gravis $(n=1)$, dissociative motor disorder $(n=2)$ and vertigo $(n=1)$. The group inflammatory neurological diseases (IND, $\mathrm{n}=33$ ) consisted of patients with cerebral systemic lupus erythematosus (SLE) $(n=14)$, post polio syndrome $(n=12)$, herpes encephalitis $(n=3)$, unspecific encephalomyelitis $(n=2)$, bacterial meningitis $(n=1)$ and cerebral vasculitis $(n=1)$. The 22 controls were analysed for various subjective conditions. However, extensive clinical and ancillary investigations have not revealed a neurological disease. The controls had normal CSF cell counts, protein and glucose levels, and normal neuroradiological examination. The local ethics committee approved the study.

The patient subgroups and patient data were coded and unknown to the investigator at the time of FACS analysis.

\section{Preparation of cell free CSF}

CSF was sampled in siliconised glass tubes. CSF samples were centrifuged at $1500 \times \mathrm{g}$ for $10 \mathrm{~min}$ at $4^{\circ} \mathrm{C}$ (to remove cells and other insoluble materials). The cell free CSF supernatant was recovered in aliquots in polypropylene tubes and stored at $-80^{\circ} \mathrm{C}$ until use.

\section{Statistical analysis}

It was technically not feasible to measure anti-myelin antibody levels in all CSF samples within one experiment. However, correlations between MFI values of CSF samples that were included in consecutive experiments were high (Pearson's Rho 0.8-1.0, $\mathrm{p}<0.001)$. Therefore, MFI values from two representative experiments were normalised and pooled for further analysis. For normalisation, regression analysis was performed on samples that were included in both experiments and the regression line was used to normalise MFI values.

Differences in anti-myelin antibody levels between several groups were analysed using the Kruskall-Wallis test. Differences 
Table 1 Patient characteristics

\begin{tabular}{lllll}
\hline Subjects (n) & $\begin{array}{l}\text { Age (years) } \\
\text { (mean (range)) }\end{array}$ & $\begin{array}{l}\text { Sex } \\
\text { (F/M) }\end{array}$ & $\begin{array}{l}\text { EDSS } \\
\text { (median (range)) }\end{array}$ & $\begin{array}{l}\text { OGB } \\
\text { (Yes/No) }\end{array}$ \\
\hline MS patients (65) & $47(19-82)$ & $41 / 24$ & $3.6(0.0-8.0)$ & $57 / 8$ \\
$\quad$ RRMS (36) & $36(19-54)$ & $24 / 12$ & $1.9(0.0-4.0)$ & $32 / 4$ \\
SPMS (29) & $54(24-82)$ & $17 / 12$ & $5.4(3.0-8.0)$ & $25 / 4$ \\
CIS patients (37) & $35(16-61)$ & $28 / 9$ & $1.5(0.0-6.5)$ & $26 / 10$ \\
HC (22)* & $40(22-61)$ & $16 / 6$ & - & $0 / 13$ \\
OND (17) & $48(25-83)$ & $8 / 9$ & - & $2 / 11$ \\
IND (33) & $51(13-75)$ & $26 / 7$ & - & $0 / 20$ \\
\hline
\end{tabular}

* $\mathrm{HC}$ are sex and age matched to the other patient groups with the exception of the OND group (sex mismatched).

$\mathrm{CIS}$, clinically isolated syndrome; EDSS, Expanded Disability Status Scale; HC, healthy controls; IND, inflammatory neurological diseases; MS, multiple sclerosis; OGB, oligoclonal bands; OND, other neurological diseases; RRMS, relapsing remitting multiple sclerosis; SPMS, secondary progressive multiple sclerosis.

between two groups were analysed by the Mann-Whitney test. Anti-myelin antibody levels in individual MS CSF samples were considered to be enhanced over control values when MFI was higher than the mean value of controls $+3 \times S D)$. Differences in the proportion of anti-myelin Ig positive samples were analysed using the $\chi^{2}$ test.

A<smiles></smiles>

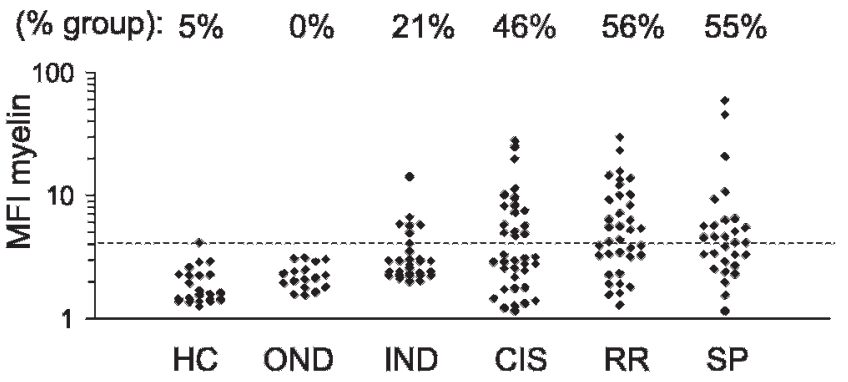

B

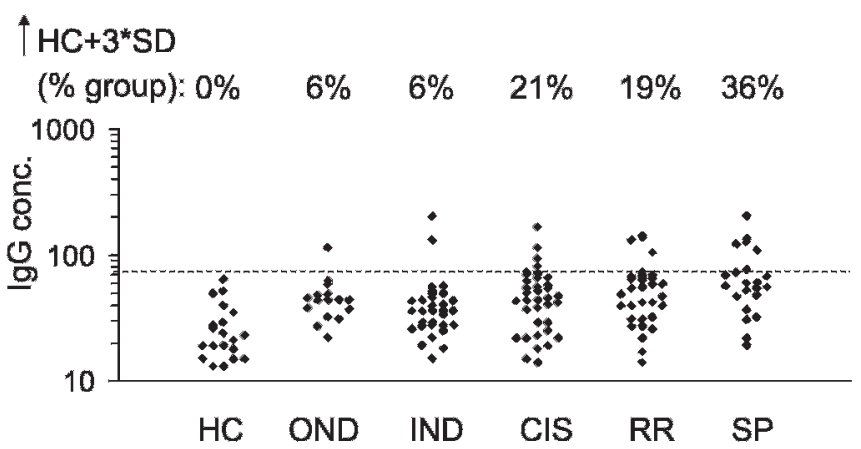

Figure 1 Increased CSF IgG antibody reactivity to myelin in patients with clinically isolated syndrome (CIS) and relapsing remitting (RRMS) or secondary progressive (SPMS) multiple sclerosis (MS). IgG antibody reactivity to myelin $(A)$ and total $\lg G$ levels $(B)$ were analysed in the CSF of controls ( $\mathrm{HC}, \mathrm{n}=22$ ), in those with other neurological diseases (OND, $\mathrm{n}=17$ ), inflammatory neurological diseases (IND, $n=33$ ), CIS patients $(n=37)$, and RRMS $(n=36)$ and SPMS $(n=29)$ patients. Myelin particles were incubated with CSF, and antibody binding to myelin was determined in each patient by secondary anti-human IgG antibodies and quantified by flow cytometry. Differences in the frequency of anti-myelinpositive patients were analysed using the $\chi^{2}$ test. Mean fluorescence intensity (MFI) differences between the patient groups were calculated using Kruskall-Wallis non-parametric analysis. The line indicates the mean MFI of $\mathrm{HC}+3 \times \mathrm{SD}$. The percentage of subjects exceeding this value is indicated above the graph.
The relationship between anti-myelin antibody levels and EDSS, disease duration and the number of T2 and gadolinium enhancing T1 lesions was determined by Spearman's correlation analysis.

A $p$ value lower than 0.05 was considered to indicate statistical significance (confidence level 95\%).

\section{RESULTS}

\section{Increased anti-myelin IgG antibody reactivity in CSF of MS patients}

To determine whether anti-myelin antibodies were present in the CSF of CIS and MS patients and patients with other neurological diseases, myelin particles were incubated with CSF samples, and binding of IgG antibodies was assessed by flow cytometry analysis. Clinical characteristics of patients and controls are summarised in table 1. Kruskall-Wallis analysis revealed significantly $(p<0.001)$ different MFI values between the several patient groups with significantly $(p<0.001)$ increased MFI values in CIS and relapse onset MS patients compared with controls and patients with other neurological diseases (fig 1A). Next, individuals were considered to have enhanced anti-myelin antibody levels if anti-myelin antibody reactivity exceeded the average of controls $+3 \times \mathrm{SD}$. The number
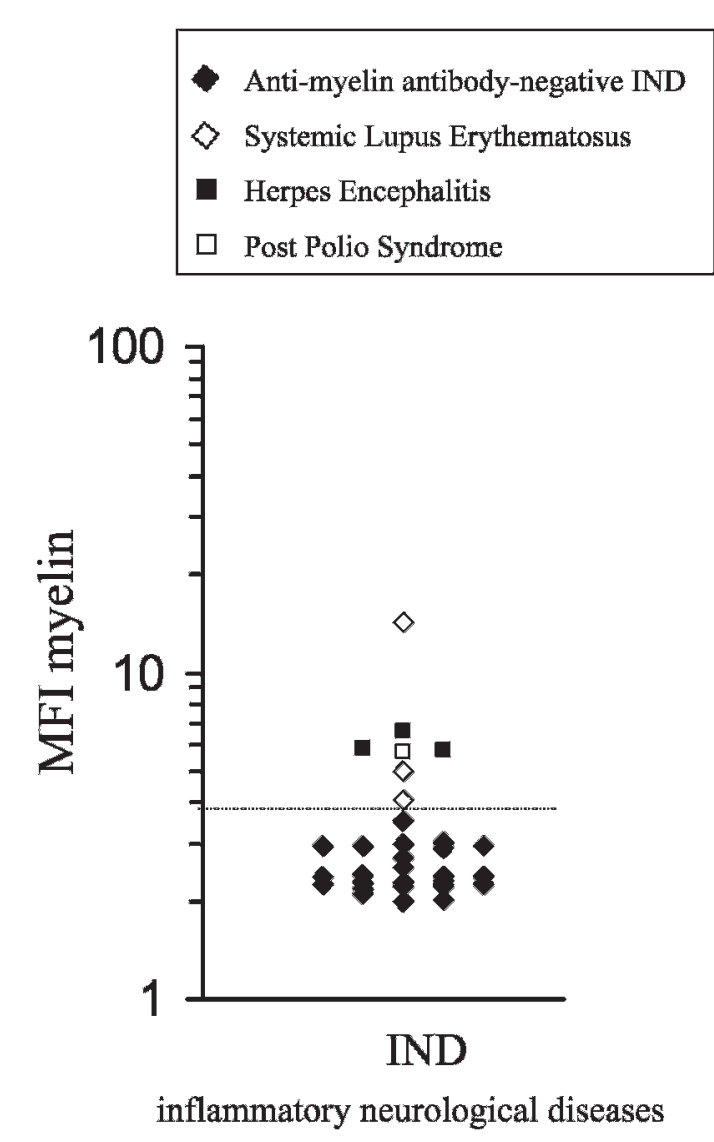

Figure 2 Increased CSF anti-myelin IgG antibody reactivity in patients with herpes encephalitis and a subpopulation of systemic lupus erythematosus (SLE) patients. CSF IgG antibody reactivity to myelin was analysed in the CSF of patients with inflammatory neurological diseases (IND, $n=33$ ). Myelin particles were incubated with CSF and antibody binding to myelin was determined in each patient by secondary antihuman IgG antibodies and quantified by flow cytometry. The line indicates the mean fluorescence intensity (MFI) of healthy controls $+3 \times$ SD. 
of patients with increased anti-myelin reactivity was significantly increased in the IND patient group (21\%, $p=0.018)$, the CIS patient group $(46 \%, p=0.002)$, and the RRMS $(56 \%$, $\mathrm{p}<0.001)$ and SPMS $(55 \%, \mathrm{p}<0.001)$ patient groups compared with controls (fig 1A). The assay demonstrated the following performance characteristics as a biomarker for diagnosis of relapse onset (RR and SP) MS: sensitivity: $52 \%$ of relapse onset MS patients exhibited elevated anti-myelin antibody reactivity; specificity: $89 \%$ of control patients (controls/OND/IND) scored negative in our assay; positive predictive value: $84 \%$ of positive CSF samples were derived from RRMS and SPMS patients.

We previously shown that monoclonal antibodies directed against the myelin proteins PLP, MBP and MOG induced a clear shift in MFI whereas incubation of isotype matched control antibodies or secondary antibodies did not induce a significant shift in MFI, suggesting that antibodies bind specifically to the myelin particles. ${ }^{22}$ As depicted in fig $1 \mathrm{~B}$, we also determined the distribution of total CSF IgG antibody levels between the several patient groups. Although Kruskall-Wallis analysis revealed that total CSF IgG levels were significantly $(p<0.001)$ different between the several patient groups, there was no significant difference between relapse onset MS patients and OND patients, which is in contrast with the MFI results. Additionally, the frequency of relapse onset MS patients with increased CSF IgG levels was not significantly different from OND patients. Normalisation of anti-myelin MFI values for IgG levels revealed a significantly elevated MFI/IgG ratio in IND $(p=0.001)$, CIS $(p=0.001)$, RRMS $(p=0.002)$ and SPMS $(p=0.006)$ patients compared with OND patients.

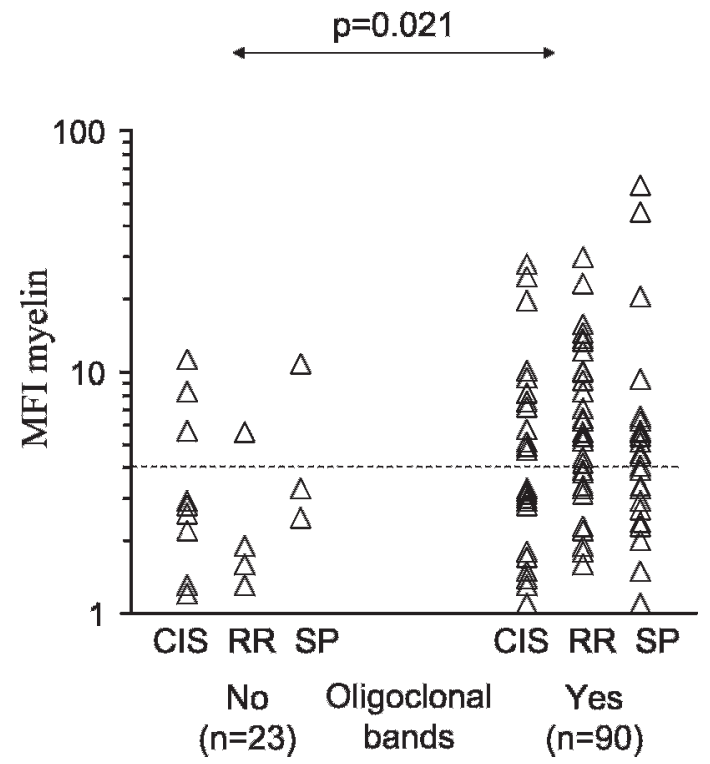

Figure 3 Increased anti-myelin IgG antibody reactivity in patients with clinically isolated syndrome (CIS) and relapsing remitting (RRMS) or secondary progressive (SPMS) multiple sclerosis (MS) with CSF oligoclonal bands. Myelin particles were incubated with CSF derived from CIS and MS patients. Antibody binding to myelin was determined in each patient by secondary anti-human IgG antibodies and quantified by flow cytometry. The difference in anti-myelin antibody reactivity between patients with (right) and without (left) oligoclonal bands was analysed using Mann-Whitney non-parametric analysis. The line indicates the mean fluorescence intensity (MFI) of healthy controls $+3 \times$ SD.
Increased anti-myelin IgG antibody reactivity in CSF of patients with CIS and IND

A substantial proportion of CIS patients (46\%, fig 1) demonstrated elevated anti-myelin antibody reactivity compared with controls. Clinical follow-up data of these patients, with a median clinical follow-up of 1 year, revealed that 12 out of 37 CIS patients developed clinically definite MS. Five out of these 12 patients (42\%) had elevated CSF anti-myelin antibody reactivity at the time of CIS diagnosis, which was very similar to the frequency observed in the entire CIS patient group. Thus these data suggest that CIS patients with a high CSF antimyelin antibody reactivity have no prevalence towards developing definite MS compared with CIS patients with low antimyelin antibody reactivity.

Anti-myelin IgG antibodies in seven out of 33 patients of the IND group exceeded the mean of controls by 3 SDs (fig 2). Three of these patients were diagnosed with cerebral SLE $(29 \%$ of entire cerebral SLE patient group). Remarkably, all three patients with herpes simplex encephalitis had increased antimyelin IgG reactivity.

\section{Increased anti-myelin IgG antibody reactivity in CIS and MS patients with CSF oligoclonal bands}

Next, we evaluated whether anti-myelin IgG antibody reactivity in CIS and MS patients was related to the presence of CSF

\section{A}
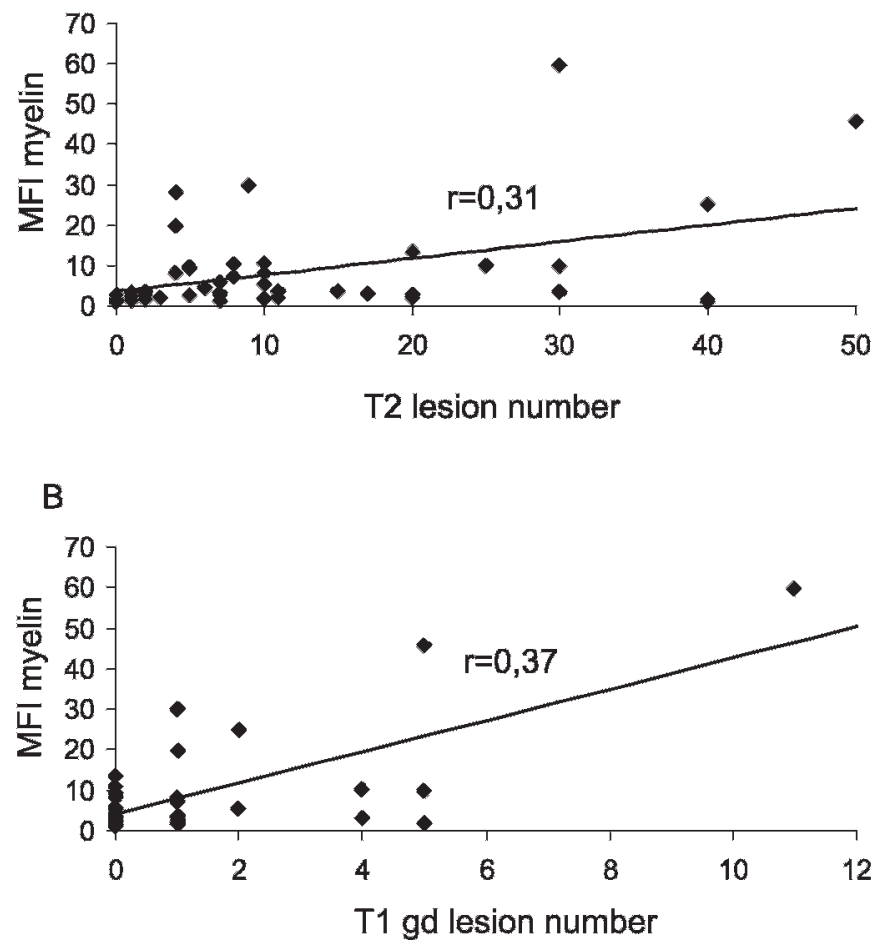

Figure 4 Significant correlation between anti-myelin IgG antibody reactivity and lesions on brain MRI. Myelin particles were incubated with CSF derived from patients with clinically isolated syndrome (CIS) and relapse onset multiple sclerosis $(n=52)$. Antibody binding to myelin was determined in each patient by secondary anti-human IgG antibodies and quantified by flow cytometry. Anti-myelin mean fluorescence intensity (MFI) values were plotted against the number of T2 lesions (A) or gadolinium (gd) enhancing T1 lesions (B) detected on brain MRI. Correlation analysis were performed using Spearman rank. 
oligoclonal bands. As depicted in fig 3, CIS and MS patients with CSF oligoclonal bands showed significantly $(p=0.021)$ increased anti-myelin antibody reactivity compared with patients without oligoclonal bands. CSF IgG levels were also significantly $(p=0.043)$ increased in patients with oligoclonal bands compared with patients without bands, whereas the $\mathrm{MFI} / \mathrm{IgG}$ ratio was not significantly different (data not shown).

\section{Anti-myelin antibody reactivity correlates with MR parameters of disease activity in CIS and MS patients}

Anti-myelin reactivity was significantly related to the number of T2 lesions $(r=0.31, p=0.041$, fig $4 A$ ). A similar significant correlation was observed between T2 lesions and CSF IgG levels $(r=0.030, p=0.043)$, whereas no significant relationship was observed between the anti-myelin MFI/IgG ratio and T2 lesions (data not shown). Anti-myelin antibody reactivity also significantly correlated with gadolinium enhancing T1 lesions $(r=0.37, p=0.016$; fig. 4B). In contrast, CSF IgG levels showed no significant correlation $(r=0.18, p=0.26)$ with $\mathrm{T} 1$ lesions, whereas the anti-myelin MFI/IgG ratio correlated significantly ( $r=0.36, p=0.020)$ with $\mathrm{T} 1$ lesions.

There was no cross sectional relation between EDSS scores or disease duration and anti-myelin IgG antibody reactivity (data not shown). In addition, we found no difference in anti-myelin antibody reactivity between RRMS patients in remission and those who underwent a relapse (data not shown).

\section{DISCUSSION}

The frequency of anti-myelin antibodies and their relation to disease activity in MS patients is controversial. This is probably because of the fact that most detection methods focus on reactivity to a single myelin protein whereas anti-myelin antibody responses in MS patients are very heterogeneous with respect to their specificities. ${ }^{21}$ Moreover, relevant antibodies may only be detected by methods that use conformational posttranslationally modified myelin epitopes whereas most methods use denaturated or recombinant myelin proteins. ${ }^{13} 1819$ Here we used a novel flow cytometry based assay that detects antibody reactivity to myelin particles, allowing the simultaneous detection of a wide variety of myelin protein and lipid specific antibodies. In addition, target epitopes are presented to the antibodies in a similar manner as they are displayed in vivo to autoreactive antibodies.

Using this method, we detected increased CSF antibody reactivity to myelin in half of the CIS and relapse onset MS patients. These results are similar to the frequency of MS patients with antibody mediated autoimmunity based on the pathology of MS lesions. ${ }^{7}$ Compared with other studies that detected antibody responses to a single myelin protein, the frequency of anti-myelin antibody positive MS patients in our study is at least twice as high. ${ }^{4}{ }^{10}$ This discrepancy may be explained by the fact that detection of antibody responses to a single myelin protein only covered part of all the different autoantibody specificities in MS patients. For instance, the recently identified myelin lipid specific antibodies are not detected by these methods. ${ }^{21}$

The frequency of anti-myelin antibody positive CIS patients that developed clinically definite MS was not different from the frequency observed in the entire CIS patient group. Although it should be noted that the median clinical follow-up in this cohort was 1 year, these results are in agreement with recent studies, showing that CIS patients with increased anti-MOG antibody reactivity have no prevalence towards developing MS compared with those without detectable anti-MOG antibodies. ${ }^{12}{ }^{15}$

We also found anti-myelin antibody reactivity in one-fifth of patients with inflammatory neurological diseases whereas nearly all controls and patients with other neurological diseases scored negative in our assay. Three out of seven positive IND patients were diagnosed for herpes encephalitis. These patients can easily be distinguished from MS patients based on clinical symptoms and specific PCR analysis. Although the number of patients is to small to draw conclusions, the coincidence of herpes encephalitis and the presence of CSF anti-myelin antibodies is interesting. Ferrante et al showed that herpes simplex virus-1 DNA and mRNA was present in a significant number of MS patients during clinical relapse. ${ }^{26}$ Other herpes viruses, such as human herpesvirus 6 and Epstein-Barr virus, have also been implicated to be associated with MS. ${ }^{27}$ Increased antibody responses to human herpesvirus 6 and Epstein-Barr virus have previously been detected in the blood and CSF of MS patients. ${ }^{28}{ }^{29}$ However, the fact that herpes infection by itself is associated with the presence of CSF anti-myelin antibodies is an interesting and novel finding. Further studies are required to investigate the coincidence of virus infections and the occurrence of CSF anti-myelin antibodies.

The detection of CSF oligoclonal IgG is currently the most useful, albeit unspecific, laboratory test to aid in the diagnosis of $\mathrm{MS}^{30}$ Oligoclonal IgG is synthesised within the CNS and is present in about $90 \%$ of patients with clinically definite MS. In this study, we demonstrated that anti-myelin antibodies were predominantly enhanced in MS patients with oligoclonal bands, indicating that oligoclonal bands may represent anti-myelin IgG antibodies. However, anti-myelin antibodies could also be detected in a substantial proportion (28\%) of the CIS and MS patients who had no CSF oligoclonal bands, suggesting that our anti-myelin antibody assay may have added value to current diagnostic CSF markers.

A number of findings suggest that different MFI values are a result of specific binding of antibodies to myelin and not just a reflection of different IgG levels. There is a clear discrepancy between the frequency of MS patients with elevated IgG levels and increased MFI values. In addition, CSF IgG levels in the OND patient group were similar to IgG levels in the IND and MS patient groups, whereas MFI values were highly different between these patient groups. Finally, increased anti-myelin antibody reactivity was associated with increased IgG levels in the relapse onset $\mathrm{MS}$ patients only $(r=0.572, p=0.001)$ whereas no correlation was observed in the OND and IND patient groups $(r=0.108(p=0.513)$, despite similar IgG levels and fluctuations.

We also demonstrated that CSF anti-myelin $\operatorname{IgG}$ antibodies were related to the number of T2 and gadolinium enhancing T1 lesions on brain MRI in CIS and relapse onset MS patients, indicating that our assay has potential to monitor disease activity (ie, active inflammation). Only limited clinical studies evaluated the relation between anti-myelin antibodies and MR measures in CIS and MS patients. Lim et al reported that antiMOG and anti-MBP antibodies in CIS patients were not associated with MRI findings at baseline or after follow-up. ${ }^{15}$ In contrast, Kuhle et al recently reported that CIS patients with anti-MOG and anti-MBP antibodies had more white matter lesions than patients without these antibodies. ${ }^{14}$ However, the majority of patients with one to nine lesions were negative for anti-MOG and anti-MBP antibodies whereas the majority of patients with comparable lesion number in our study were antibody positive. Thus our assay may detect anti-myelin 
antibody responses at an early stage of the disease whereas antiMOG and anti-MBP responses may develop during later stages of the disease.

Further studies in paired serum/CSF samples have to be performed to evaluate whether the increased CSF anti-myelin antibody reactivity is intrathecally produced. Additionally, it remains to be elucidated whether the autoreactive anti-myelin antibodies contribute to the pathogenesis of MS. Our findings that increased anti-myelin antibody reactivity in CSF of relapse onset MS patients correlate with disease activity at an early stage of the disease are in agreement with a potential role of these antibodies in pathogenesis of MS. Alternatively, antimyelin antibodies may merely represent an epiphenomenon that develops in response to continuous myelin breakdown. However, regardless of whether anti-myelin antibodies are causative or not, we clearly demonstrated that anti-myelin antibodies, detected with myelin particles, have potential as specific and promising biomarkers for MS disease activity.

Funding: This study was financially supported by the Netherlands Foundation for MS Research.

Competing interests: None.

Ethics approval: The study was approved by the local ethics committee.

\section{REFERENCES}

1. Steinman L, Martin R, Bernard C, et al. Multiple sclerosis: deeper understanding of its pathogenesis reveals new targets for therapy. Annu Rev Neurosci 2002;25:491-505.

2. Genain CP, Cannella B, Hauser SL, et al. Identification of autoantibodies associated with myelin damage in multiple sclerosis. Nat Med 1999;5:170-5.

3. Schluesener HJ, Sobel RA, Linington $\mathrm{C}$, et al. A monoclonal antibody against a myelin oligodendrocyte glycoprotein induces relapses and demyelination in central nervous system autoimmune disease. J Immunol 1987;139:4016-21.

4. Zhou D, Srivastava R, Nessler S, et al. Identification of a pathogenic antibody response to native myelin oligodendrocyte glycoprotein in multiple sclerosis. Proc Natl Acad Sci U S A 2006:103:19057-62.

5. Brosnan CF, Raine CS. Mechanisms of immune injury in multiple sclerosis. Brain Pathol 1996;6:243-57.

6. Brink BP, Veerhuis R, Breij EC, et al. The pathology of multiple sclerosis is locationdependent: no significant complement activation is detected in purely cortical lesions. J Neuropathol Exp Neurol 2005;64:147-55.

7. Lucchinetti C, Bruck W, Parisi J, et al. Heterogeneity of multiple sclerosis lesions: implications for the pathogenesis of demyelination. Ann Neurol 2000;47:707-17.

8. Keegan M, Konig F, McClelland R, et al. Relation between humoral pathological changes in multiple sclerosis and response to therapeutic plasma exchange. Lancet 2005;366:579-82.

9. Reindl M, Linington C, Brehm U, et al. Antibodies against the myelin oligodendrocyte glycoprotein and the myelin basic protein in multiple sclerosis and other neurological diseases: a comparative study. Brain 1999;122:2047-56.
10. Karni A, Bakimer-Kleiner R, Abramsky 0, et al. Elevated levels of antibody to myelin oligodendrocyte glycoprotein is not specific for patients with multiple sclerosis. Arch Neurol 1999;56:311-15.

11. O'Connor KC, McLaughlin KA, De Jager PL, et al. Self-antigen tetramers discriminate between myelin autoantibodies to native or denatured protein. Nat Med 2007:13:211-17.

12. Kuhle J, Pohl C, Mehling M, et al. Lack of association between antimyelin antibodies and progression to multiple sclerosis. N Engl J Med 2007;356:371-8.

13. Berger T, Rubner P, Schautzer F, et al. Antimyelin antibodies as a predictor of clinically definite multiple sclerosis after a first demyelinating event. N Engl J Med 2003;349:139-45

14. Kuhle J, Lindberg RL, Regeniter A, et al. Antimyelin antibodies in clinically isolated syndromes correlate with inflammation in MRI and CSF. J Neurol 2007;254:160-8.

15. Lim ET, Berger T, Reindl M, et al. Anti-myelin antibodies do not allow earlier diagnosis of multiple sclerosis. Mult Scler 2005;11:492-4.

16. von Budingen HC, Hauser SL, Ouallet JC, et al. Frontline: Epitope recognition on the myelin/oligodendrocyte glycoprotein differentially influences disease phenotype and antibody effector functions in autoimmune demyelination. Eur $\mathrm{J}$ Immunol 2004;34:2072-83.

17. O'connor KC, Appel H, Bregoli L, et al. Antibodies from inflamed central nervous system tissue recognize myelin oligodendrocyte glycoprotein. J Immunol 2005; 175:1974-82.

18. Haase CG, Guggenmos J, Brehm U, et al. The fine specificity of the myelin oligodendrocyte glycoprotein autoantibody response in patients with multiple sclerosis and normal healthy controls. J Neuroimmunol 2001;114:220-5.

19. Mesleh MF, Belmar N, Lu CW, et al. Marmoset fine B cell and T cell epitope specificities mapped onto a homology model of the extracellular domain of human myelin oligodendrocyte glycoprotein. Neurobiol Dis 2002;9:160-72.

20. Carotenuto A, D'Ursi AM, Nardi E, et al. Conformational analysis of a glycosylated human myelin oligodendrocyte glycoprotein peptide epitope able to detect antibody response in multiple sclerosis. J Med Chem 2001;44:2378-81.

21. Kanter JL, Narayana S, Ho PP, et al. Lipid microarrays identify key mediators of autoimmune brain inflammation. Nat Med 2006;12:138-43.

22. Breij EC, Heijnen $\mathrm{P}$, van der GA, et al. Myelin flow cytometry assay detects enhanced levels of antibodies to human whole myelin in a subpopulation of multiple sclerosis patients. J Neuroimmunol 2006;176:106-14.

23. van der Goes A, Kortekaas M, Hoekstra K, et al. The role of anti-myelin (auto)antibodies in the phagocytosis of myelin by macrophages. J Neuroimmunol 1999;101:61-7.

24. McDonald WI, Compston A, Edan G, et al. Recommended diagnostic criteria for multiple sclerosis: guidelines from the International Panel on the diagnosis of multiple sclerosis. Ann Neurol 2001;50:121-7.

25. Lublin FD, Reingold SC. Defining the clinical course of multiple sclerosis: results of an international survey. National Multiple Sclerosis Society (USA) Advisory Committee on Clinical Trials of New Agents in Multiple Sclerosis. Neurology 1996;46:907-11.

26. Ferrante $\mathbf{P}$, Mancuso R, Pagani E, et al. Molecular evidences for a role of HSV-1 in multiple sclerosis clinical acute attack. J Neurovirol 2000;6(Suppl 2):S109-14.

27. Gilden DH. Infectious causes of multiple sclerosis. Lancet Neurol 2005:4:195-202

28. Soldan SS, Berti R, Salem N, et al. Association of human herpes virus 6 (HHV-6) with multiple sclerosis: increased IgM response to HHV-6 early antigen and detection of serum HHV-6 DNA. Nat Med 1997:3:1394-7.

29. Bray PF, Luka J, Bray PF, et al. Antibodies against Epstein-Barr nuclear antigen (EBNA) in multiple sclerosis CSF, and two pentapeptide sequence identities between EBNA and myelin basic protein. Neurology 1992;42:1798-804.

30. Link H, Huang YM. Oligoclonal bands in multiple sclerosis cerebrospinal fluid: an update on methodology and clinical usefulness. J Neuroimmunol 2006:180:17-28. 